Recuperación de trabajo de fin de grado

\title{
Políticas Institucionales contra las Violencias Machistas
}

\section{Análisis de caso Protocolo en la FCPolit}

Institutional Policies against Sexist Violence.

Protocol case analysis at FCPOLIT

Georgina Santervas

Licenciada en Ciencia Política

(Universidad Nacional de Rosario)

Investigadora en Centro de Investigaciones

Feministas y Estudios de Género

(Universidad Nacional de Rosario)

Correo: santervasgeorgina@gmail.com 


\title{
Resumen
}

El presente artículo es un fragmento de la tesina de grado en la que nos propusimos analizar las políticas institucionales contra las violencias machistas, a través del caso concreto del Procedimiento para la Atención de la Violencia de Género, de la Facultad de Ciencia Política y Relaciones Internacionales (U.N.R.). Específicamente se aborda el estudio de las violencias machistas en el marco de la educación superior, identificando sus particularidades y revisando cómo fue construido el problema público y la estructuración de esta política. La comprensión del proceso de recontextualización en el que se enmarca el Procedimiento para la Atención de la Violencia de Género, abre un panorama de análisis crítico de las políticas públicas frente a las demandas sociales que se presentan a partir de la irrupción de la cuarta ola feminista en los espacios públicos.

\section{Palabras clave}

Violencias sexistas, Universidad, Políticas públicas, Feminismos.

\begin{abstract}
This article is a fragment of the degree thesis in which we set out to analyze institutional policies against sexist violence, through the specific case of the Procedure for the Attention of Gender Violence, of the Faculty of Political Science and International Relations. Specifically, the study of sexist violence in the context of higher education is addressed, identifying its particularities and reviewing how the public problem was constructed and the structuring of this policy. The understanding of the process of recontextualization in which the Procedure for the Attention of Gender Violence is framed, opens a panorama of critical analysis of public policies against the social demands that arise from the emergence of the fourth feminist wave In public spaces.
\end{abstract}

\section{Keywords}

Sexist violence, University, Public policies, Feminisms. 


\section{Introducción}

El presente artículo tiene por objetivo exponer de manera resumida la tesina de grado para la finalización de la Licenciatura en Ciencia Política (UNR). Dicha tesina estuvo motivada por la coyuntura socio-político que nos toca transitar y protagonizar, a partir de la irrupción masiva del movimiento feminista en la escena pública para transformar la realidad y politizar nuestra cotidianidad. Las reivindicaciones son claras y contundentes a partir del primer Ni Una Menos, la masificación de la lucha de la campaña por el Aborto Legal, Seguro y Gratuito, y los Paros Internacionales de Mujeres que llevaron a una mundialización de las vivencias y saberes de las mujeres trabajadoras. Todo ello marca un clima de época, que nos interpela e invita a realizar apuestas teóricas en pos de revolucionar también la academia.

Retomamos las posturas de la epistemología feminista que valoriza la adopción y explicitación del punto de vista de quien investiga, esto implica situarse para transformar la experiencia en saber (Dorlin, 2009). La pretensión de neutralidad, desde esta perspectiva, implica negar la historia y la opresión social; por eso, apostamos a retomar las experiencias de las mujeres, identidades feminizadas y disidencias sexuales, esclareciendo las visiones del sujeto cognoscente.

El saber feminista se apoya en todo un conjunto de saberes locales, de saberes diferenciales y oposicionales, descalificados, considerados como 'incapaces de unanimidad' o 'no conceptuales', que tiene que ver con la reapropiación de sí: de su cuerpo, de su identidad (Dorlin, 2009:15).

En este sentido, la propuesta de analizar el Procedimiento para la Atención de la Violencia de Género ${ }^{1}$, el Acoso sexual y la Discriminación basada en el Género, Orientación sexual, Identidad de género o expresión de género de la Facultad de Ciencia Política y Relaciones Internacionales, de la Universidad Nacional de Rosario (UNR), cuyo dispositivo de atención comenzó

1 "Violencias de Género" en adelante serán abreviadas como "VdG". 
a funcionar en el año 2015, se centra en caracterizar las violencias sexistas en el marco de la Educación Superior, y sistematizar las acciones y líneas de trabajo que se desarrollaron desde el Procedimiento para la atención, la prevención y sensibilización de $\mathrm{VdG}$.

\section{La Universidad no es la excepción}

El carácter estructural de las violencias sexistas, y las asimetrías de poder que generan, se manifiestan en todos los ámbitos de nuestras vidas. Las instituciones en las cuales desarrollamos nuestras tareas cotidianas, como la Universidad, no permanecen ajenas a esta construcción de vinculaciones y entramados organizacionales.

Analizar las violencias sexistas en los ámbitos educativos y laborales permite tensionar los mitos de domesticidad y la reducción a las formas de violencias físicas de las violencias sexistas, para así comprenderlas como relaciones de poder constitutivas de nuestras subjetividades, deseos y formas de transitar las instituciones, en este caso académicas. Esos mitos también construyen imaginarios clasistas, que exceptúan y excepcionalizan las violencias ejercidas por sujetes de la clase media con acceso a la universidad (Rovetto y Figueroa, 2017). A su vez, establecen formas "adecuadas" para las víctimas, que tienen que demostrar determinadas características como vulnerabilidad, debilidad y escasos lazos de contención.

La invisibilización de las manifestaciones cotidianas de las violencias sexistas en los ámbitos de estudio y trabajo, mediados a su vez por las prácticas de producción y reproducción de conocimientos, y la determinación de cuáles son los conocimientos legítimos, generan un entramado de poder que tiene un anclaje importante en las desigualdades sexo-género.

Una de las formas de violencias sexistas más frecuentes en los ámbitos de la educación superior es el acoso. Raunigg y Cristoffanini (2019) en su investigación sobre violencias de género en las universidades chilenas, enmarcan este tipo de violencias de la siguiente forma: "el acoso sexual es violencia de género porque es uno de los reflejos de la desigualdad estructural, material y simbólica que vivenciamos las mujeres que vivimos en sociedades patriarcales" (2019:235). 
A su vez, Rovetto y Figueroa (2017) destacan que puede identificarse como fenómenos articulados con la discriminación a partir de la masiva incorporación de las mujeres en las Universidades Nacionales. Las autoras resaltan que este tipo de prácticas agresivas exceden las posibilidades de visualizarlo, lo que dificulta su nominación, más allá del registro de sentirnos mal e incómodas cuando se produce. "Sin embargo, quienes lo padecen en el ámbito universitario, tienden a no denunciar porque se sobreentiende que es así como funcionan las cosas" (Rovetto y Figueroa, 2017:3).

Retomando a Raunigg y Cristoffanini (2019) es que podemos identificar algunas tipologías de acoso en los ámbitos de la educación superior. Una es el chantaje sexual o el acoso quid pro quo ${ }^{2}$, en este tipo de acoso tiene una íntima vinculación con las posiciones de poder y jerarquía. Una segunda tipología es la de acoso ambiental, situaciones de violencias donde quien acosa genera un entorno hostil, humillante e intimidatorio. Una tercera categoría es el acoso sexista, que comprende hostigamiento en el cual queda de manifiesto el lugar de desprecio, valor secundario e incapacidad en el que la persona violenta coloca a las mujeres y disidencias.

En Argentina son pocos los estudios que dan cuenta de la dimensión del problema del acoso en los espacios universitarios. Sin embargo, es preciso resaltar la importancia de explorar cualitativa y cuantitativamente -de ser posible- las manifestaciones de las violencias sexistas en estos ámbitos. En términos de Rafael Blanco:

La vida cotidiana en las universidades argentinas, y especialmente el modo cómo el paso por éstas modula las biografías de numerosos jóvenes en el marco de una época signada por las transformaciones de los órdenes públicos, privado e íntimo, constituye un terreno de reflexión poco explorado. Sin embargo, atender a este fenómeno permite profundizar en la comprensión de las universidades no sólo como instituciones de transmisión de conocimiento sino también como instituciones que intervienen activamente en la producción de subjetividades (2014:56).

2 Del latín "esto a cambio de eso". Para más información consultar Raunigg y Cristoffanini (2019) 
Morgade, Decana de Filosofía y Letras de la Universidad Nacional de Buenos Aires (UBA) destaca que "lo que los feminismos y los movimientos de la disidencia sexual han estado mostrando es que estos procesos de violencia, de discriminación, o de segregación también se producen dentro de las universidades" (2019: párr. 6). En este sentido, las universidades en tanto espacio educativo no pueden dejarlos pasar, ya que en ellas se están formando futurxs profesionales de distintos campos disciplinares, "y la ética de los derechos humanos y la ética del respeto por las identidades sexo genéricas y la ética antiviolencia, son parte de una ética central en la formación de profesionales en las universidades" (Morgade, 2019, párr. 6).

Las relaciones de poder propias de las estructuras educativas y laborales, cobran en el marco de la Universidad una significancia particular, ya que están mediadas por formas de conocer, saberes jerarquizados por la academia, o por estructuras de poder manifiestas como las relaciones docentes-estudiantes. Estas particularidades en las formas de vinculaciones en los espacios de educación superior, se manifiestan claramente en el inconsciente colectivo de que las "cosas funcionan así” y que la autoridad que se adquiere por "méritos" académicos permite un lugar que, en apariencia, es intocable.

Si bien es cierto estadísticamente, que actualmente hay una enorme cantidad de mujeres en las aulas, eso no cambia las prácticas cotidianas, ni modifica las relaciones de poder o los métodos de enseñanza (Morgade, 2019). Sin embargo, gracias a la labor constante e incansable de las feministas en la academia, se comienza a incorporar una perspectiva que estuvo durante siglos ausente de los campos de producción de conocimiento, lo que también habla de las lógicas hegemónicas de validar los saberes en un sistema androcéntrico y eurocéntrico. Morgade señala: "sabemos que no es suficiente con que haya muchas mujeres, pero sabemos que muchas mujeres con una mirada feminista también tensan a la investigación y tensan al trabajo en la universidad, por eso es importante que haya mujeres" (2019: párr. 4).

En este sentido, la disputa no es sólo por reconocer los lugares fronterizos que históricamente ocupamos las mujeres y nuestros saberes en la academia, sino tensionar y ocupar todos los canales que se abren a partir de este movimiento masivo de mujeres en las calles y en las Universidades, para 
modificar la forma en la que se transmiten los saberes y cuáles son jerárquicamente valorados.

Los procesos de identificación y reconocimiento de las estructuras silenciosas de las violencias sexistas simbólicas, y en paralelo idear formas novedosas de canalizar las nuevas experiencias de denuncia, con el eje político de cambiar las instituciones, tienden a "mover los cimientos patriarcales de la institución universitaria” (Vázquez Laba y Rugna, 2015:110).

La centralidad de desentramar (e idear políticas públicas tendientes a ello) las violencias sexistas en el ámbito universitario radica en comenzar a echar luz sobre aquellas violencias simbólicas, que como dijimos anteriormente, son la base material de la dominación patriarcal. Las situaciones de riesgo a las que las mujeres, identidades feminizadas y disidencias nos enfrentamos a diario, tienen como telón de fondo lugares y momentos que no son necesariamente considerados "de riesgo". Esto nos posiciona en un rol de constante auto defensa, que muchas veces resulta instintiva, ya que muchos de estos mecanismos son naturalizados por la forma en la que los tenemos aprehendidos.

Siempre he pensado que hablar de estos temas en nuestro ambiente laboral es como hablar de la violencia doméstica, conyugal o el incesto en las familias, la mayoría de los miembros saben de su existencia e incluso conocen quién o quiénes son las personas que ejercen violencia pero nadie denuncia los hechos (Huacuz Elías, 2016:32).

Siguiendo este planteo es que retomamos la noción de "derecho a no saber" desarrollada por Migno y Moreno (2015), mediante la cual las autoras identifican la persistencia de individuos privilegiados, en el universo de la educación superior, que recurren a la "opción de no saber" para ignorar los beneficios que obtienen de sus relaciones, su lugar de dominio y su reafirmación de inocencia en el ejercicio sistemático de hábitos opresivos. Este derecho a no saber según las autoras, se basa en una ignorancia que es menos cognitiva que performativa, es decir no se trata de la simple carencia de información, sino de la elección propia de no reconocer dicha información por la implicación subjetiva en la misma: 
Uno de los procesos que se desencadenan a partir del cultivo de la ignorancia acerca de la violencia de género, y en particular en lo concerniente al acoso sexual, es el silencio que de diversas formas se impone o se fomenta en las mujeres que son objeto de esta práctica (Migno y Moreno, 2015:140).

Al cruce entre "el derecho a no saber", el reconocimiento establecido de que "las cosas son asî", tiene un tercer componente que responde a la dificultad de las mujeres de clase media, universitarias, a las que se le puede añadir recorridos militantes o lugares profesionales con fuerte carga política, de asumirnos víctimas (Figueroa y Rovetto, 2017). Es decir, afrontar que todas las teorías que desarrollamos y explicamos, nos pasan.

Las formas en las que fuimos socializadas, también implican un cuidado de otrxs por encima de nuestro bienestar, por lo que, a la hora de denunciar este tipo de violencias, nos lleva a un análisis de costos/beneficios en los que generalmente se minimizan y postergan las denuncias o el accionar colectivo sobre las violencias sexistas.

Develar las tramas invisibilizadas de violencias en nuestra vida cotidiana (violencia simbólica, psicológica, institucional, acoso sexual, entre otras) se da en un proceso determinado para nuestras sociedades, donde las formas de violencias más explícitas (femicidios, travesticidios, violaciones) ya adquirieron, producto de la movilización y el trabajo de numerosas mujeres y disidencias, una visibilización fundamental.

En este marco, los esfuerzos de quienes le ponen el cuerpo en las instituciones académicas, están siendo materializados a través de nuevos marcos regulatorios y nuevas instancias de discusión y abordaje de la perspectiva de género en estas instituciones educativas (Rovetto y Figueroa, 2019). 


\title{
La política del Protocolo de género en la FCPOLIT
}

\author{
Si hay algo novedoso en materia de vida universitaria es \\ la aparición de los protocolos para atender las violencias \\ ejercidas especialmente contra las mujeres
}

Dora Barrancos, 2019

Hasta el año 2014 la única Universidad Nacional que contaba con un protocolo específico para abordar la violencia de género era la Universidad Nacional del Comahue, que comprende las provincias patagónicas de Neuquén y Río Negro. A su vez, en septiembre de 2015, con el fin de fortalecer el proceso colectivamente, se crea la "Red Interuniversitaria por la igualdad de género y contra las violencias" 3 .

Concretamente en la Facultad de Ciencia Política y Relaciones Internacionales (UNR), se aprueba el procedimiento a fines de 2014, siendo una de las unidades académicas a la vanguardia en este tipo de política, mediante la Resolución de Consejo Directivo ${ }^{4}$ N$^{\circ} 2573 / 14^{5}$, elaborada e impulsada por docentes, graduadas e investigadoras, con el fin de visibilizar la problemática de las violencias sexistas, conformar un espacio de atención y constituir un marco regulatorio a través de políticas institucionales.

En palabras de Florencia Rovetto, una de las propulsoras del proyecto: "Hay violencias simbólicas atravesadas por situaciones de género, de poder, y hacía falta un instrumento como este, encuadrado en la Convención de Belém do Pará como marco jurídico sobre violencia de género y acoso sexual" (2014: parr. 3). La intención del proyecto es la de brindar asesoramiento legal y asegurar un ámbito de privacidad que evite la revictimización, para todxs lxs que se acerquen a consultar y/o denunciar. El objetivo es erradicar

3 La red interuniversitaria por la igualdad de género y contra las violencias, se gestó como idea en las XII Jornadas de Historia de las Mujeres/ VII Congreso de Estudios de género (en marzo de 2015 en Neuquén) y se concretó el 3 de septiembre de ese mismo año en la Universidad Nacional de San Martín con la presencia de feministas de 25 universidades argentinas.

4 Órgano de co-gobierno de la Facultad, integrado por docentes, nodocentes, estudiantes, graduades y el decano de la institución.

5 Res. CD. N²573/14 "Procedimiento para la Atención de la Violencia de género, el acoso sexual y la discriminación de género en la Facultad de Ciencia Política y Relaciones Internacionales de la Universidad nacional de Rosario". 
y desnaturalizar la violencia de género, la más sutil de las violencias, concluyó Rovetto en dicha entrevista. (Rovetto, 2014).

Estas concepciones jurídico-normativas estructuran una forma de concebir a la política pública por parte de la institución educativa, generando el acceso a procesos oportunos que resguarden los derechos, la intimidad, y eviten la revictimización. La generación de políticas institucionales que aborden integralmente la problemática de las violencias sexistas en el transcurrir cotidiano por las universidades, contribuye a construir prácticas políticas en materia de sensibilización, prevención y erradicación de las violencias sexistas. Estos marcos normativos amplían y efectivizan derechos, a la vez que generan un compromiso institucional de abordar situaciones de violencias sexistas que afectan las libertades fundamentales de quienes estudian y/o trabajan en los espacios universitarios.

Cabe resaltar que desde la perspectiva que se abordan las violencias sexistas en el marco del procedimiento para la atención de VdG en la Facultad de Ciencia Política, se da un giro que lleva a la política más allá de la escucha e intervención “después del hecho”. Las líneas de acción de sensibilización, transversalización de la perspectiva de género y la concreción de espacios de escucha y problematización colectiva, tienden a la idea y práctica de lo que Elsa Dorlin denomina autodefensa “adoptar un giro más francamente muscular: sinceramente creo que tenemos que volver a los cuerpos, al poder que reside dentro nuestro; usar nuestro enojo, bronca, sentido de justicia, desesperación, y convertirlos en poder de auto-defensa" (Dorlin, 2019: párr. 5).

Los abordajes feministas y la estructuración de políticas públicas en esta perspectiva, generan no sólo medidas protectivas, sino también reparatorias, reconociendo los derechos vulnerados que han sido históricamente soslayados. La construcción de lazos comunitarios, el abordaje de políticas de lo común, implican un compromiso ético por parte de la institución educativa. "La enunciación es herramienta y, a la vez, bandera de lucha que tensiona el secretismo patriarcal que subyace bajo cada agresión sexista" (Raunigg y Cristoffanini, 2019:234).

Es posible pensar estas nuevas políticas con perspectiva de género en las Universidades Nacionales como prácticas activas de feminización de la 
política, retomando el concepto desarrollado por la jurista española María Eugenia Palop (2019). Esto implica, reivindicar las formas de la política que ponen a los vínculos relacionales en el centro, comprendiendo que nuestros proyectos personales dependen de nuestra capacidad de (re)conocer y (re) construir relaciones. Esta perspectiva nos permite pensar las políticas de redes que desde los feminismos se plantean enmarcadas en las vivencias de las interrelaciones y las conexiones emocionales.

Este giro de feminización de la política, tiene en las Universidades un primer paso evidente con la aprobación de diversos protocolos y procedimientos de actuación ante las violencias sexistas, que habilitan nuevas e innovadoras prácticas que mueven los cimientos patriarcales de la academia, y que nos permiten imaginar nuevas formas de hacer política universitaria.

\section{Abordajes}

Habiendo abordado aspectos relativos a la conceptualización en el marco del dispositivo de atención, cabe problematizar las primeras experiencias y lineamientos de trabajo que moldearon, y continúan haciéndolo, al procedimiento en tanto política situada en un entramado de constantes cambios y reconfiguraciones. Las violencias machistas, como fueron planteadas en el proceso de elaboración de la política institucional, no pueden ser tratadas de una única manera, ni de una vez y para siempre, reconocer esto implica la mutación de una política pública cimentada en "arenas movedizas".

Precisamente estas consideraciones son las que llevaron a que el Procedimiento para la atención de VdG se estructure con dos ejes de acción diferenciados, por un lado las líneas de trabajo relacionadas con la sensibilización y la prevención, que tiene como objetivo fundamental visibilizar la problemática en el ámbito universitario, y que a su vez genera las condiciones para que la política sea apropiada por los distintos claustros que constituyen la población de la Facultad, facilitando la argumentación con el objetivo de adquirir legitimidad y reconocimiento.

Por otro lado, los lineamientos y desafíos en torno a las estrategias de intervención directa, de atención, escucha y acompañamiento de casos que fueron abordados en estos cinco años de actuación del Protocolo de género. 
Si bien entendemos que resulta limitado analizar una política sólo a través de información cuantificable, consideramos que el análisis de esto datos concretos, nos permitirá expresar de manera gráfica lo expuesto anteriormente; poniendo en valor el trabajo realizado y aportando a las posteriores investigaciones que profundicen sobre las especificidades de las violencias sexistas en las Universidades y a la conformación de nuevos marcos regulatorios.

\section{Los desafíos de la atención}

En lo que refiere específicamente al espacio de atención, el mismo está conformado solo por una persona responsable, seleccionada a través de concurso público y abierto. Los canales de comunicación se encuentran especificados en la página web de la Facultad, estableciendo que la metodología para la concreción de un primer encuentro es a través de la coordinación vía mail. Las demandas al espacio fueron variando desde su implementación, ya que en un inicio la Fcpolit era una de las primeras unidades académicas en contar con un espacio de atención a $\mathrm{VdG}$, por lo que se recepcionaron consultas que no necesariamente involucran a personas de dicha facultad.

En este apartado nos abocaremos a analizar la información que se desprende de estos primeros años de atención, acompañamiento y escucha de casos. Cabe mencionar que evaluar de qué forma y en qué medidas las metas logran alcanzarse es una tarea que no puede reducirse a un recuento mensurable; sin embargo, la agregación y el análisis de algunas percepciones aportan datos que también resultan útiles a la hora de balancear desempeños, revisar y reorientar la política del procedimiento de atención.

La recolección y el análisis de los datos, logra transferir de forma organizada las intervenciones concretas del Protocolo de actuación contra VdG a partir de 2015 hasta mediados de 2019. El análisis cuantitativo y cualitativo sistematiza la información, pone en valor el trabajo realizado, permitiendo visualizar con claridad que ésta es una tarea necesaria en pos de democratizar e igualar aún más las condiciones dentro de las Universidades Públicas. Cabe destacar que quienes realizan este tipo de trabajos son mujeres comprometidas, lo que no implica que su labor quede singularizada a una 
práctica militante, donde nuevamente se pone el foco en el amor y la responsabilidad asumida a priori.

Las experiencias concretas sobre las que se trabajó desde el Procedimiento de Atención en los cinco años de funcionamiento (2015 a 2019) arrojan información cuantitativa que permite reflexionar sobre las prácticas de abordaje en la educación superior, confirmando que la Universidad no está exenta de situaciones de violencia y discriminación basadas en la identidad de género u orientación sexual, y que sus repercusiones afectan la forma de habitar los espacios institucionales, la vida académica, profesional y laboral. Cabe destacar que un proceso de sistematización de datos como este, es el primero de estas características dentro de los Espacios de Atención de VdG que actualmente están funcionando en las Facultades de la UNR.

\section{Análisis de casos}

Como se ha mencionado, el Procedimiento para la atención se efectiviza en el año 2015. A partir de entonces, hasta agosto de 2019, se ha intervenido en un total de $22 \operatorname{casos}^{6}$ notando un incremento acelerado de las denuncias en el segundo año (2016) demostrando la eficacia de las actividades, dispositivos y herramientas de sensibilización y difusión desarrolladas por espacio de atención. En el año 2018 se registran más denuncias que en los años anteriores, en parte puede deberse a la visibilización y mediatización de la problemática y casos resonantes, que durante ese año tomaron dimensiones inéditas.

En relación al género de las personas que han denunciado casi en su totalidad fueron mujeres, salvo una excepción. Las denuncias provienen en su mayoría del claustro estudiantil, lo que refleja la apropiación por parte de las mujeres más jóvenes de esta herramienta institucional, sin embargo, durante los últimos dos años aquí analizados (2018 y 2019) se presentaron

6 Cabe mencionar que los datos aquí analizados responden al registro de acompañamientos y denuncias que cumplen con las condiciones de pertinencia establecidas por el Procedimiento en su marco regulatorio, sin embargo, el espacio de atención de la Fcpolit recibió consultas que si bien fueron acompañadas y asesoradas, no corresponden a lo especificado por la resolución de consejo directivo, por lo que no están consideradas dentro de este análisis cuantitativo, a fin de no distorsionar la información. 
dos denuncias por parte de mujeres de los otros dos claustros, docente y no docente, lo que nos permite pensar en un principio de acercamiento del espacio con las mujeres que desarrollan estas tareas.

En lo que respecta a las personas denunciadas, en su mayoría se trata de varones, salvo una excepción. Aquí se diversifica más el claustro al que pertenecen: encontramos que el 13,6\% son docentes, el 9,1\% no docentes y el $77,3 \%$ son estudiantes. De este último grupo existen denunciados con más de una denuncia.

Tal como resaltan numerosas feministas "lo que no se nombra no existe" por lo que es de suma importancia asignarles categorías concretas a las violencias machistas que las mujeres y disidencias, que transitamos la vida universitaria en diferentes claustros, estamos comenzando a visualizar. Como mencionamos en los apartados precedentes, la dificultad de la nominación, más allá del registro de "sentirnos mal" (Rovetto y Figueroa, 2017) es una de las principales dificultades con las que nos encontramos cuando las violencias machistas no se manifiestan en sus formas más brutales.

El trabajo que se realiza en el espacio de atención implica, entre otras tantas cuestiones, ponerle título a esas violencias que sentimos, pero no logramos nombrar. Entre los casos aquí analizados, vemos la prevalencia de la violencia verbal, psicológica y emocional (34,48\%) y de las manifestaciones de acoso sexual $(27,58 \%)$. Muchas de las denuncias tienen más de una forma de violencia machista, lo que amplifica la idea de que las violencias se expresan de diversas maneras, y es necesario advertirlas desde las manifestaciones más leves para poder prevenir sus caras más cruentas.

Aquí desarrollamos la violencia psicológica, entendida como "acciones intencionadas que conllevan un daño y/o riesgo para la integridad psíquica de la víctima. Se manifiesta de múltiples formas: insultos, humillaciones, amenazas, etc., que son expresadas abierta o sutilmente" (Gipuzkoa Foru Aldundia, 2014:19). Este tipo en particular, conlleva una gran dificultad para advertirla, ya que en general sus efectos no son percibidos de inmediato, más bien el deterioro es subjetivo: pérdida de autoestima, autovaloración, percepción de riesgo, entre otros. Es fundamental reconocer que cuando existe cualquier otro tipo de violencia, también está presente la violencia 
psicológica. La violencia física se refiere a "acciones de carácter intencional que conllevan daño y/o riesgo para la integridad física" (2014:19). Por su parte, la violencia sexual se refiere a "acciones que obligan a una persona a mantener intimidad sexual forzada (por intimidación, coacción u otro mecanismo que anule o limite la voluntad personal)" (2014:20) y particularmente el acoso sexual implica comportamientos "verbales, no verbales y físicos de índole sexual y no deseados, (...) con el propósito o el efecto de atentar contra la dignidad de la mujer y/o de crear un entorno intimidatorio hostil, degradante, humillante u ofensivo" (2014:31) ${ }^{7}$.

A partir de los datos recabados y sistematizados en este análisis de casos, podemos inferir que somos las mujeres jóvenes las que nos apropiamos de las herramientas institucionales, de los canales de denuncia y de las diversas propuestas tendientes a visibilizar, sensibilizar y prevenir las violencias machistas en el marco de la Facultad de Ciencia Política y Relaciones Internacionales. De igual manera, es importante reconocer los impulsos de aquellas mujeres (en su mayoría docentes, graduadas y funcionarias) que motorizan este tipo de políticas universitarias. Pero, fundamentalmente, el reconocimiento es para las académicas feministas que desde sus militancias, investigaciones y trabajo constante habilitaron e impulsaron esta reforma en el modo de comprender la academia.

Específicamente respecto al espacio de atención, se puede señalar un creciente reconocimiento del mismo en el aumento en el número de denuncias, y un inicio de diversificación con respecto al claustro denunciante, lo que responde, como se viene señalado, a la dificultad de las mujeres con determinadas características de reconocer que estas violencias también nos tienen a nosotras al frente de batalla. Sin embargo, hasta el momento todas las personas denunciantes (salvo una excepción) se auto perciben como mujeres, lo que implica analizar mecanismos para que otras identidades subalternizadas puedan nombrar las violencias que pueden llegar a percibir.

7 Existen múltiples formas de violencias machistas que no fueron desarrolladas aquí, por no contar con casos registrados por el procedimiento, como las vinculadas con la homofobia, la lesbofobia, la transfobia y una multiplicidad de violencias sexuales. 
Por último, podemos hipotetizar que las formas que adquieren estas violencias machistas pueden diversificarse aún más, a través de mecanismos específicos, para continuar con la dominación basada en patrones sexo-genéricos, pero también por causas ajenas, como puede ser el uso de redes sociales y nuevos canales de comunicación entre los miembros de la comunidad educativa.

\section{Reflexiones finales}

A modo de cierre de este trabajo de investigación, nos proponemos realizar algunas reflexiones finales, que lejos de concluir con la temática desarrollada en estas páginas, nos abre un panorama de nuevos interrogantes y perspectivas de abordajes futuras.

Como mencionamos en la introducción a este trabajo, el mismo se inscribe en una coyuntura signada por el protagonismo de las mujeres latinoamericanas en las calles de nuestros países; por lo que los ámbitos educativos y académicos no pueden quedar exentos de la multiplicidad de cambios que el movimiento feminista está demandando.

Por tanto, la apuesta política de estas páginas se propuso ser un aporte más a las investigaciones recientes de numerosas mujeres, donde la perspectiva de género es el motor fundamental que transforma las miradas y metodologías. Entendemos que el camino no está despojado de conflictos, es por esto que consideramos imperioso reconocer y otorgarle un lugar central en nuestras prácticas académicas y públicas, que ponen especial atención a las relaciones de poder y de dominación patriarcal en las instituciones donde pasamos gran parte de nuestras vidas, nos formamos y (con)formamos subjetividades, y a su vez, donde se desarrollan perspectivas teóricas que determinan saberes hegemónicos.

Este artículo responde a un interés político colectivo de continuar empapando a la Universidad de las transformaciones populares. Que la Universidad Pública sea popular y feminista, no puede ser sólo una consigna, tiene que ser la quimera que nos movilice día a día a transformarnos y transformar las formas en las que conocemos, las maneras de vincularnos, los saberes que valoramos y la amplitud de nuestras casas de estudios. 
Una nueva Reforma Universitaria está dispuesta a revolucionar nuestras realidades. Las políticas públicas en el marco de la universidad son la clave latente de que estamos transitando un momento convulsionado, que nos implica un trabajo constante, tiempo, definición política, y la convicción de que venimos a transformar cada uno de los ámbitos de nuestras vidas.

\section{Referencias bibliográficas}

Biglia, Bárbara (2005). Narrativas de mujeres sobre las relaciones de género en los Movimientos Sociales. (Programa de doctorado) La representación mental: Cognició, Comunicaciói Ciencia. Universidad de Barcelona, Barcelona, España.

Biglia, Bárbara (2007). "Resignificando violencia(s): obra feminista en tres actos y un falso epílogo". En B Biglia y C San Martín (Ed.), Estado de wonderbra (pp. 21-34). Barcelona, España, Virus Editorial

Biglia, Bárbara y San Martín, Conchi (2007). Estado de wonderbra. Entretejiendo narraciones feministas sobre las violencias de género. Barcelona, España, Virus Editorial.

Blanco, Rafael (2014). Normatividades de la política cotidiana. Género y sexualidad en los saberes, la política y la sociabilidad universitaria. Propuesta educativa, 2(42), año 23.

Carmona, Lluïsa (2003). A, ante, abajo, con, contra, de, desde... Diez años aprendiendo de nosotras, las mujeres. Duoda. Revista de Estudios Feministas, (24), 192-200.

Cruz, Verónica y Vasquez, Eliana (2018). "Violencia de Género y Universidad. Hacia la consolidación de una política de reconocimiento y ampliación de derechos. En P. Rojo y V. Jardon (Ed.), Los enfoques de género en las universidades (pp. 110-120). Universidad Nacional de Rosario, Argentina. Recuperado de: http://grupomontevideo.org/sitio/wp-content/ uploads/2018/11/Libro-AUGM-2018-5.pdf

Dorlin, Elsa (7 de junio de 2019). Cuando digo no es no Suplemento / Entrevistada por Yuszczuk. Las12, Página 12. Recuperado de: https://www.pagina12.com.ar/198536-cuandodigo-no-es-no

Femenías, María Luisa (2013). Violencias Cotidianas, en las vidas de las mujeres. Rosario, Argentina, Prohistoria Ediciones.

Gebruers, Cecilia (2012). Acoso sexual en espacios educativos en Argentina. Una aproximación a su regulación y abordaje a partir de la revisión de decisiones judiciales. Documento de Trabajo ELA. Buenos Aires, Argentina.

Gipuzkoa Foru Aldundia (2014). Documento sobre la violencia machista, la terminología utilizada por la diputación foral de Gipurkoa y el análisis que la sustenta. Cataluña, España.

Huacuz Elías, María Guadalupe (2016). "Recuperar la investigación acción: estrategias de resistencia a la violencia en los espacios universitarios. El caso del "Programa institucional Cuerpos que importan en la Universidad Autónoma Metropolitana Unidad Xochimilco". En F. Boccardi; A. Boria, y C. Harrington (Ed.), Genealogías de la violencia (pp. 27-48). Córdoba, Argentina, Centro de Estudios Avanzados. Recuperado de: https://rdu.unc.edu.ar/ 
bitstream/handle/11086/4779/Genealogias $\% 20$ de $\% 201$ a $\% 20$ violencia $\% 20 \% 20$ Digital.pdf? sequence $=1 \&$ is Allowed $=\mathrm{y}$

Ley 26.743: Ley de Identidad de Género. Boletín Oficial de la Nación Argentina (24 de mayo de 2012) Recuperado de: http://servicios.infoleg.gob.ar/infolegInternet/anexos/195000-199999/197860/norma.htm

Ley 26485: Ley de protección integral para prevenir, sancionar y erradicar la violencia contra las mujeres en los ámbitos donde desarrollen sus relaciones interpersonales. Boletín Oficial de la Nación Argentina (8 de mayo de 2019) Recuperado de: http://servicios.infoleg.gob.ar/ infolegInternet/anexos/150000-154999/152155/norma.htm

Ley 27499: Ley Micaela de capacitación obligatoria en género para todas las personas que integran los tres poderes del Estado. Boletín Oficial de la Nación Argentina (10 de enero de 2019) Recuperado de: http://servicios.infoleg.gob.ar/infolegInternet/anexos/315000-319999/318666/norma.htm

López, María Pía (2019). Inventamos o erramos. Suplemento Las 12. Página 12. Recuperado de: https://www.pagina12.com.ar/177733-inventamos-o-erramos

Martínez, Margarita (2019). El reflejo de resistir. Filosofía de la violencia. Revista Anfibia. Recuperado de: http://revistaanfibia.com/ensayo/el-reflejo-de-resistir/

Morgade, Graciela (2019). Las universidades como territorio del patriarcado / Entrevistada por Bonsignore. Página 12. Recuperado de: https://www.pagina12.com.ar/206500-las-universidades-como-territorio-del-patriarcado

Ordenanza $\mathrm{N}^{\circ}$ 734: Protocolo de actuación ante situaciones de violencia y discriminación de género. Universidad Nacional de Rosario (Exp. N74415/7D) Rosario, Argentina (5 de junio de 2018)

Ramírez Raunigg, Karin y Trujillo Cristoffanini, Macarena (2019). Acoso sexual como violencia de género: voces y experiencias universitarias chilena. Cuestiones de género: de la igualdad y la diferencia, (14), 221-240. http://revpubli.unileon.es/ojs/index.php/cuestionesdegenero/article/download/5779/4477

Resolución de Consejo Directivo N 2573/14 "Procedimiento para la atención de la violencia de género, el acoso sexual y la discriminación de género en la Facultad de Ciencia Política y Relaciones Internacionales de la Universidad Nacional de Rosario Facultad de Ciencia Política y Relaciones Internacionales, Universidad Nacional de Rosario, Rosario, Argentina (2014)

Rovetto, Florencia (9 de noviembre de 2014). Casos en la UNR. Suplemento rosario12. Página 12. Recuperado de: https://www.pagina12.com.ar/diario/suplementos/rosario/9-46625-2014-11-09.html

Rovetto, Florencia y Figueroa Noelia (2017). Que la universidad se pinte de feminismos para enfrentar las violencias sexistas. Descentrada, 1(2). https://rephip.unr.edu.ar/handle/2133/9029

Rovetto, Florencia y Figueroa Noelia (2019 a). Universidad pública feminista: desafíos para eliminar las violencias sexistas en la educación superior. Texto inédito.

Rovetto, Florencia y Figueroa Noelia (2019 b). Abordajes feministas frente a las violencias sexistas en la Universidad. Texto inédito. 
Vazquez Laba, Vanesa (2016). La lucha es en el campus, el claustro y el pasillo. Revista Anfibia. Recuperado de: http://revistaanfibia.com/la-lucha-es-en-el-campus-el-claustro-y-el-pasillo/

Vázquez Laba, Vanesa y Rugna, Cecilia. (2015) Aulas sin violencia, Universidades sin violencia. La experiencia del Programa contra Violencia de Género de la Universidad Nacional de San Martín. Revista del IICE (38), 109-118. https://doi.org/10.34096/riice.n38.3466

Vázquez Laba, Vanesa; Palumbo, Mariana. y Fernández, Carla (2016) ¿Cómo prevenir, sancionar y erradicar la violencia de género en las Universidades? Universidad Nacional de Buenos Aires. Recuperado de: http://www.sociales.uba.ar/wp-content/blogs.dir/219/files/2016/11/19.-dossier-FERNANDEZ.pdf

Recibido: 27/03/2020

Aceptado: 18/11/2020 